\title{
Supporting Information - Flexible Bidentate Pyridine and Chiral Ligands in the Self-Assembly of Supramolecular 3-D Cages
}

Ki-Whan Chi, ${ }^{\dagger}$ Chris Addicott, ${ }^{*}$ Yury K. Kryschenko, and Peter J. Stang*

Department of Chemistry, University of Utah, 315 S. 1400 E. Salt Lake City, Utah 84112

${ }^{\dagger}$ Visiting Professor from the Department of Chemistry, University of Ulsan, Ulsan 680-

749 Republic of Korea.

stang@chem.utah.edu

\section{Table of Contents}

Synthesis of pyridyl tripod $\mathbf{1 c}-\mathbf{d}$.

Figure S1. ${ }^{31} \mathrm{P}$ NMR of $\mathbf{3 a}$.

Figure S2. ${ }^{1} \mathrm{H}$ NMR of $\mathbf{3 a}$.

Figure S3. ESI-MS of 3a.

Figure $\mathrm{S} 4 .{ }^{31} \mathrm{P}$ NMR of $\mathbf{3 b}$.

Figure S5. ${ }^{1} \mathrm{H}$ NMR of $\mathbf{3 b}$.

Figure S6. ESI-MS of $\mathbf{3 b}$.

Figure S7. ${ }^{31} \mathrm{P}$ NMR of $\mathbf{3 c}$.

Figure S8. ${ }^{1} \mathrm{H}$ NMR of $\mathbf{3 c}$.

Figure S9. ESI-MS of $\mathbf{3 c}$.

Figure S10. ${ }^{31} \mathrm{P}$ NMR of $\mathbf{3 d}$.

Figure S11. ${ }^{1} \mathrm{H}$ NMR of $\mathbf{3 d}$.

Figure S12. ESI-MS of $\mathbf{3 d}$.

Figure S13. ${ }^{31} \mathrm{P}$ NMR of $\mathbf{3 e}-\mathbf{f}$.

Figure S14. ${ }^{1} \mathrm{H}$ NMR of $\mathbf{3 e}-\mathbf{f}$.

Figure S15. ESI-MS of $\mathbf{3 e}$. 
General: Tris(4-pyridyl)methanol 4 was prepared from 4-iodopyridine ${ }^{1}$ and ethyl isonicotinate (Acros) using modified conditions to those reported. ${ }^{2}$ 2-Phenylbutyryl chloride 5 was prepared from the corresponding enantiomer of 2-phenylbutyric acid (Aldrich) as described. ${ }^{3}$

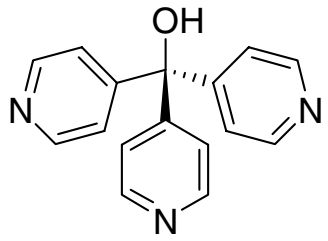

4<smiles>CCC(C(=O)Cl)c1ccccc1</smiles>

5a: $R$

5b: $\mathrm{S}$

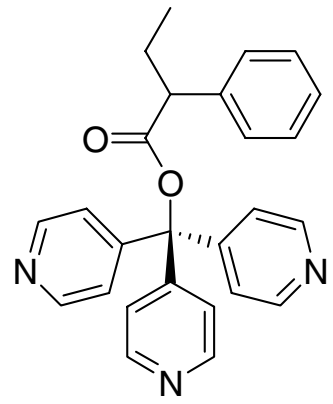

1c: $R$

Scheme S1. Synthesis of chiral tripods $1 \mathbf{c}-\mathbf{d}$.

Synthesis of 1c - d: Tris(4-pyridyl)methanol 4 (250mg, 0.95mmol) and THF were added to an oven-dried 3-necked flask under $\mathrm{N}_{2}$. The reaction was cooled to $-85{ }^{\circ} \mathrm{C}$ in an acetone / dry ice / liquid nitrogen bath. n-Butyllithium $(0.42 \mathrm{ml}$ of a $2.5 \mathrm{M}$ solution in hexanes, $1.05 \mathrm{mmol}$ ) was added slowly to the stirred mixture. The reaction was warmed to $-30{ }^{\circ} \mathrm{C}$ then cooled back to $-85{ }^{\circ} \mathrm{C}$. 2-Phenylbutyryl chloride 5 (182mg, $\left.1 \mathrm{mmol}\right)$ was added and the reaction was then warmed to room temperature overnight. The solvent was removed in vacuo and the residue chromatographed on silica gel $(5 \%$ methanol / acetone). The product $\mathbf{1 c}-\mathbf{d}$ was initially obtained as a yellow oil. Addition of an ethyl acetate / hexane mixture (3ml, approximately 1:3) and ultrasound treatment (5 minutes) afforded $1 \mathbf{c}-\mathbf{d}$ as a white solid, which was collected and washed with hexane $(2 \times 2 \mathrm{ml})$ then dried under $\mathrm{N}_{2}$. Yield $=123 \mathrm{mg}, 32 \%$ of $\mathbf{1 c} ; 111 \mathrm{mg}, 28 \%$ of 1d. M.p. $=172-174$ ${ }^{\circ} \mathrm{C} .[\alpha]_{\mathrm{D}}=-31.8(\mathbf{1 c}),+33.5(\mathbf{1 d}) ;[\phi]_{\mathrm{D}}=-130(\mathbf{1 c}),+137(\mathbf{1 d})$. 


\footnotetext{
${ }^{1} \mathrm{H}$ NMR $\left(\mathrm{CD}_{3} \mathrm{OD}, 300 \mathrm{MHz}\right) \delta 8.48\left(\mathrm{~d},{ }^{3} \mathrm{~J} 6.11 \mathrm{~Hz}, 6 \mathrm{H}, \mathrm{H}_{\alpha-\mathrm{Py}}\right), 7.31\left(\mathrm{~m}, 11 \mathrm{H}, \mathrm{H}_{\beta}-\mathrm{Py}\right.$ and $\left.\mathrm{H}_{\text {phenyl }}\right), 3.83$ (t, $\left.{ }^{3} \mathrm{~J} 7.67 \mathrm{~Hz}, 1 \mathrm{H}, \mathrm{C}(\mathrm{O})-\mathrm{CH}\right), 2.06(\mathrm{~m}, 1 \mathrm{H}, \mathrm{C}(\mathrm{O})-\mathrm{CH}(\mathrm{Ph})-\mathrm{CH}), 1.79$ (m, $1 \mathrm{H}, \mathrm{C}(\mathrm{O})-\mathrm{CH}(\mathrm{Ph})-\mathrm{CH} H), 0.85\left(\mathrm{t},{ }^{3} \mathrm{~J} 7.37 \mathrm{~Hz}, 3 \mathrm{H},-\mathrm{CH}_{3}\right)$.
}

\section{References}

(1) Coudret, C. Synth. commun. 1996, 26, 3543.

(2) Fujita, M.; Yu, S.-Y.; Kusukawa, T.; Funaki, H.; Ogura, K.; Yamaguchi, K. Angew. Chem. Int. Ed. 1998, 37, 2082.

(3) Jones, P. R.; Goller, E. J.; Kauffman, W. J. J. Org. Chem. 1971, 36, 3311. 


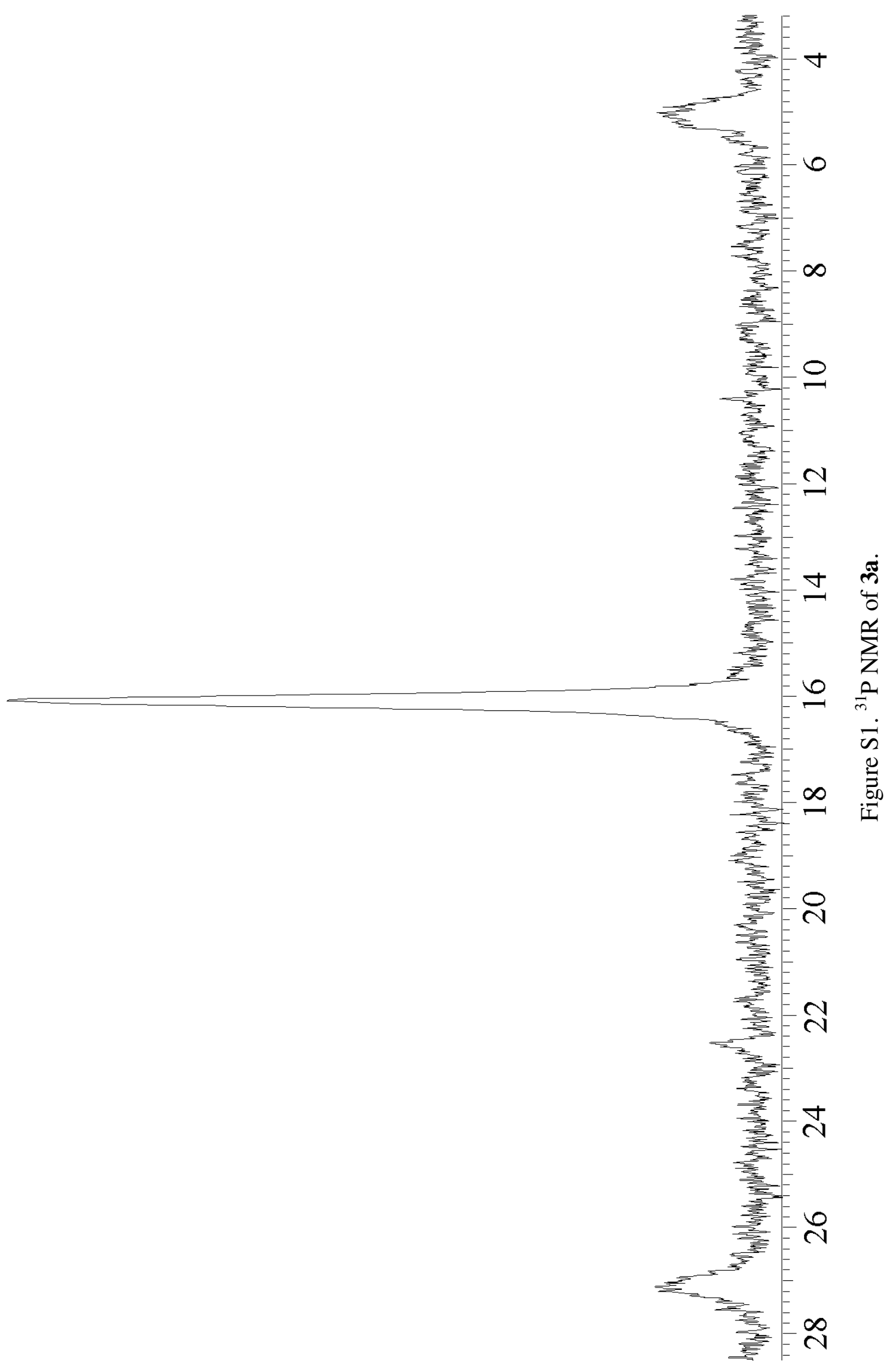














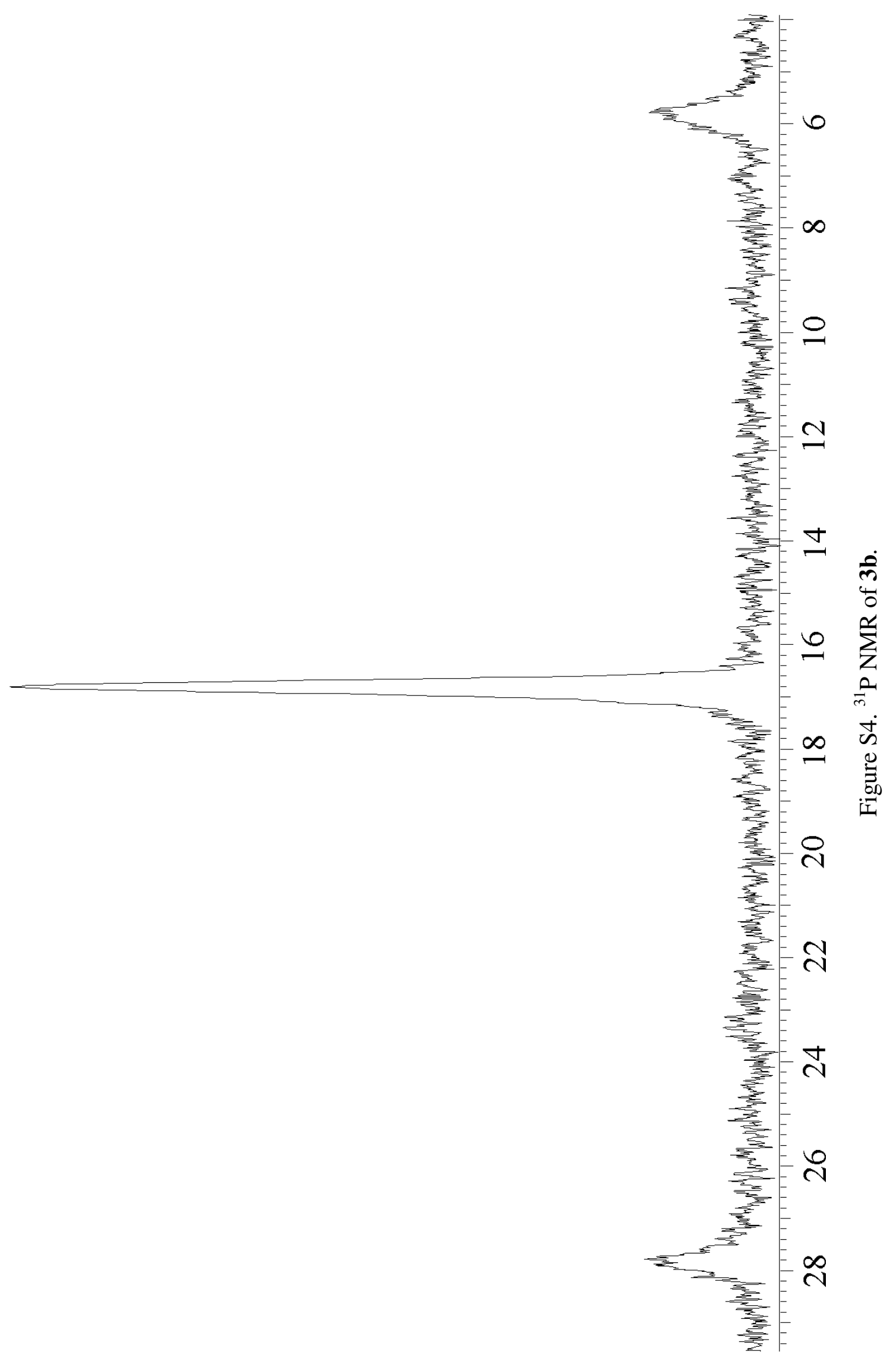

v 


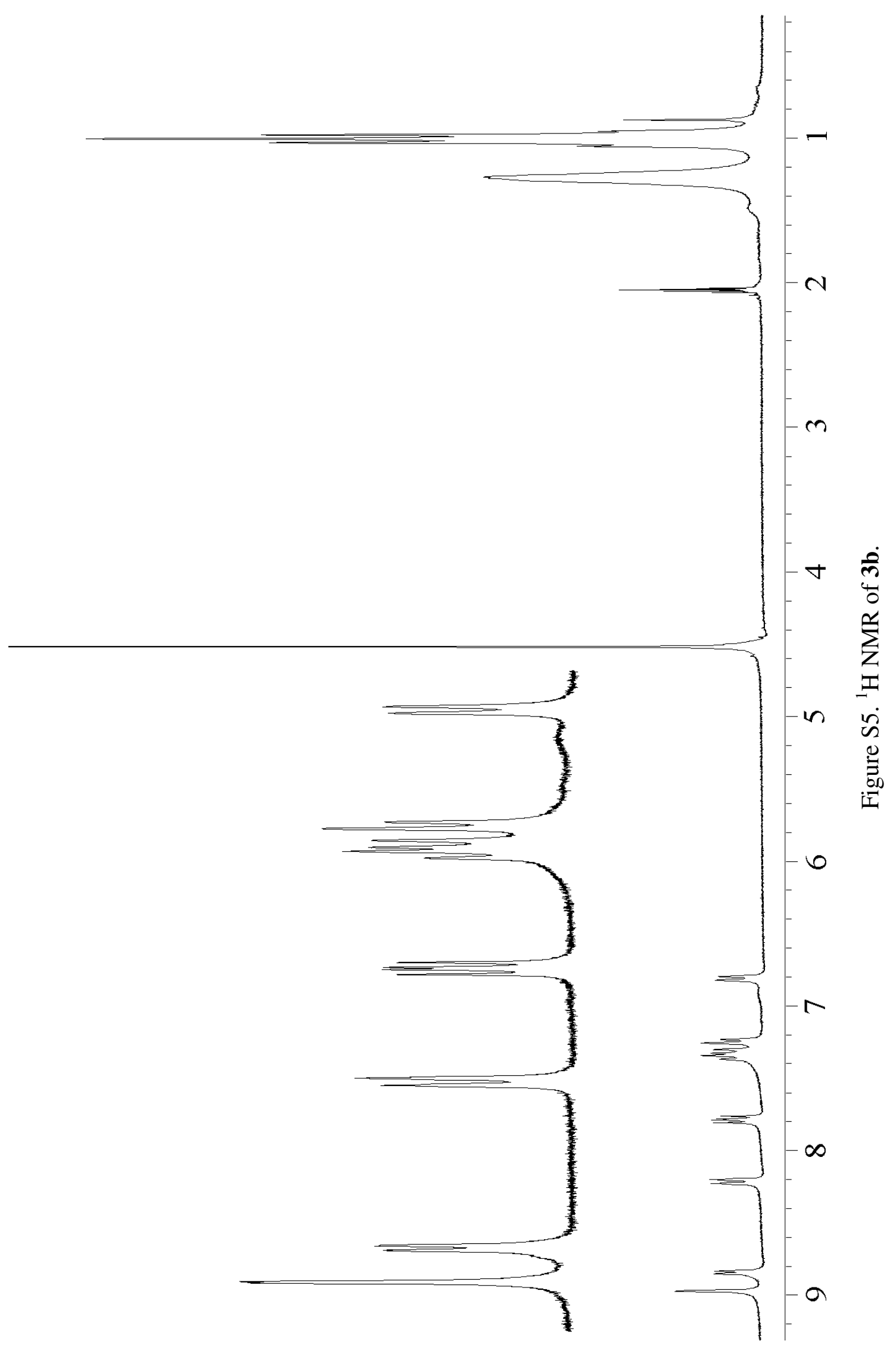




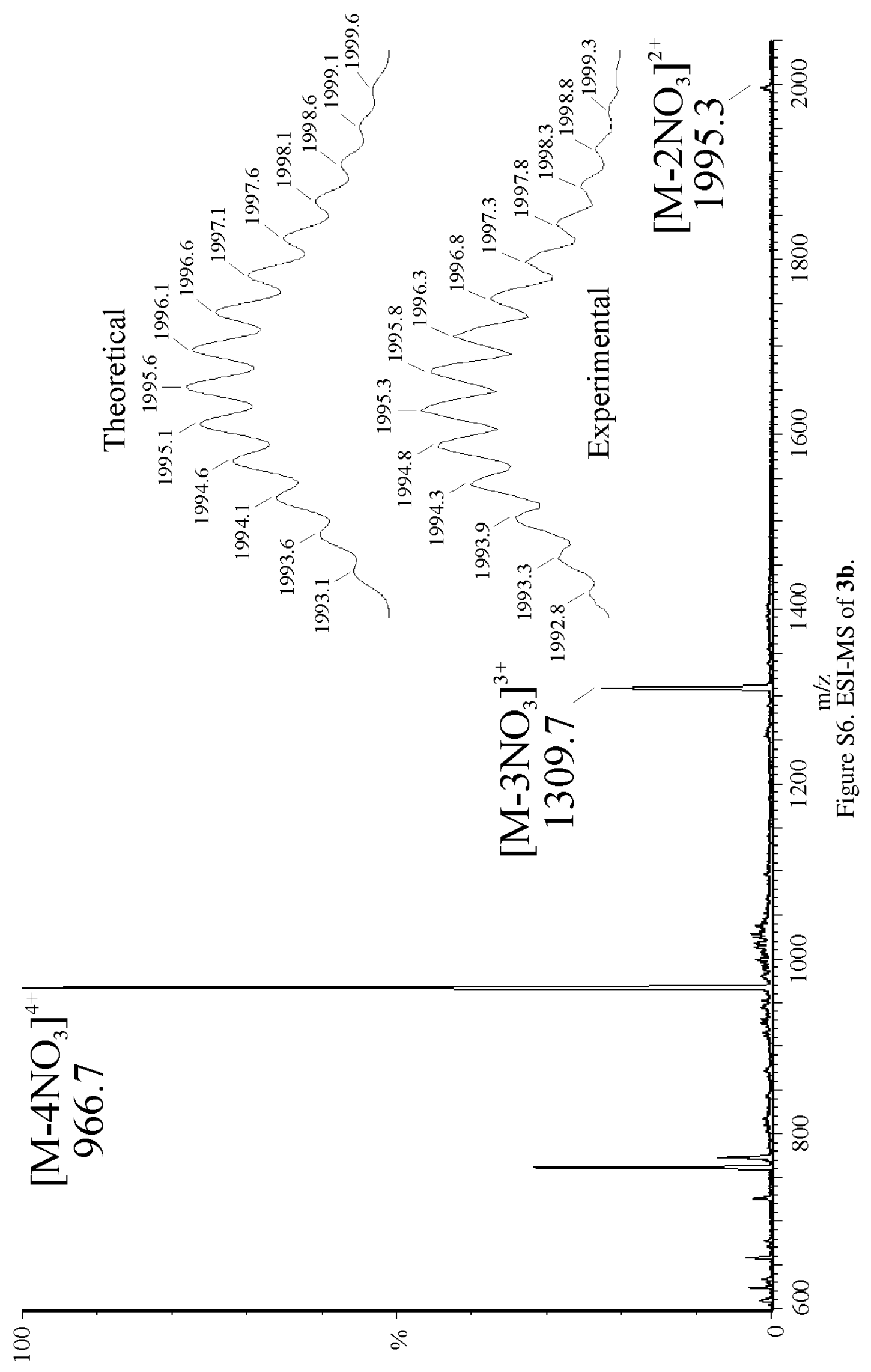

is 


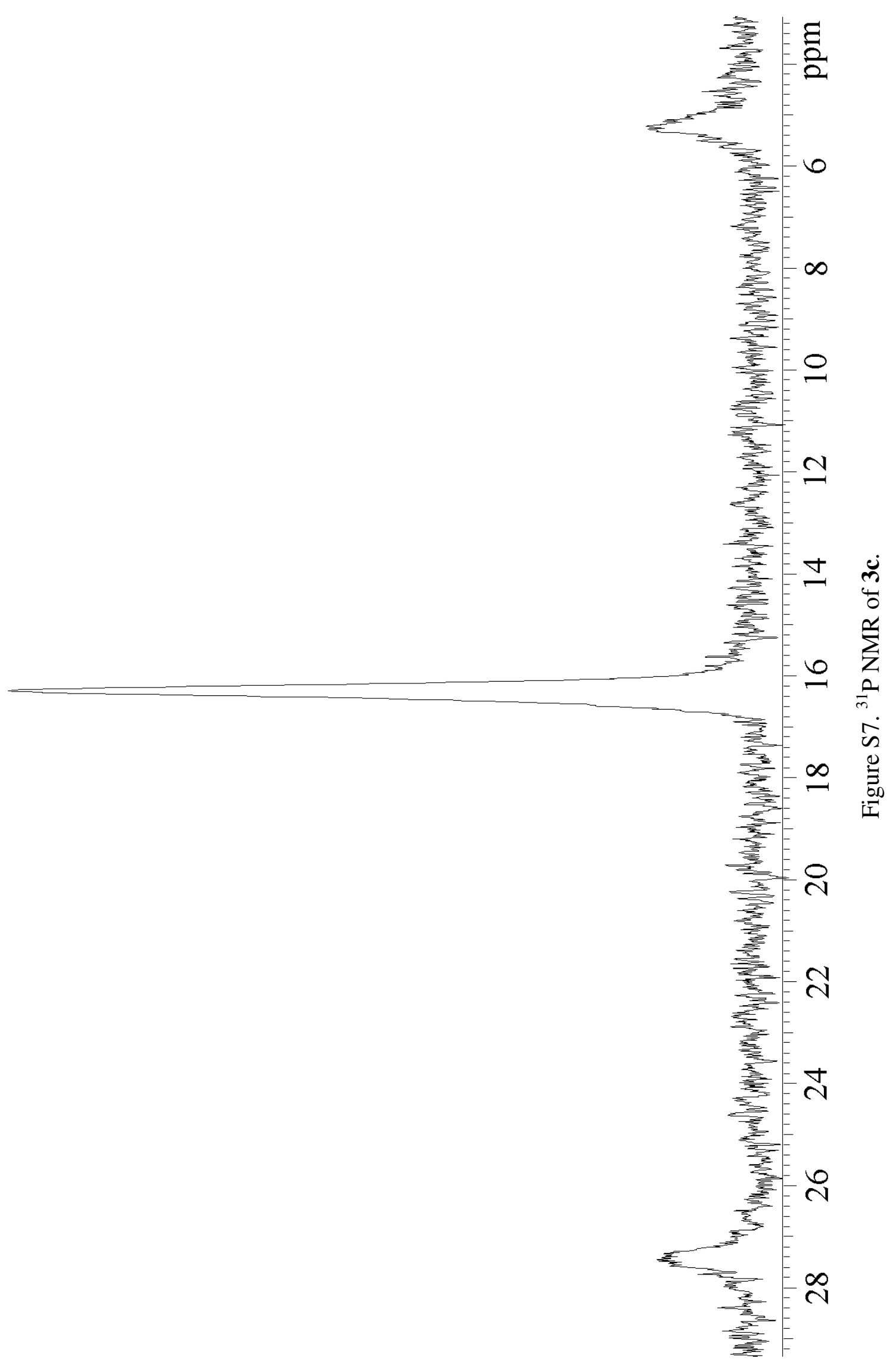




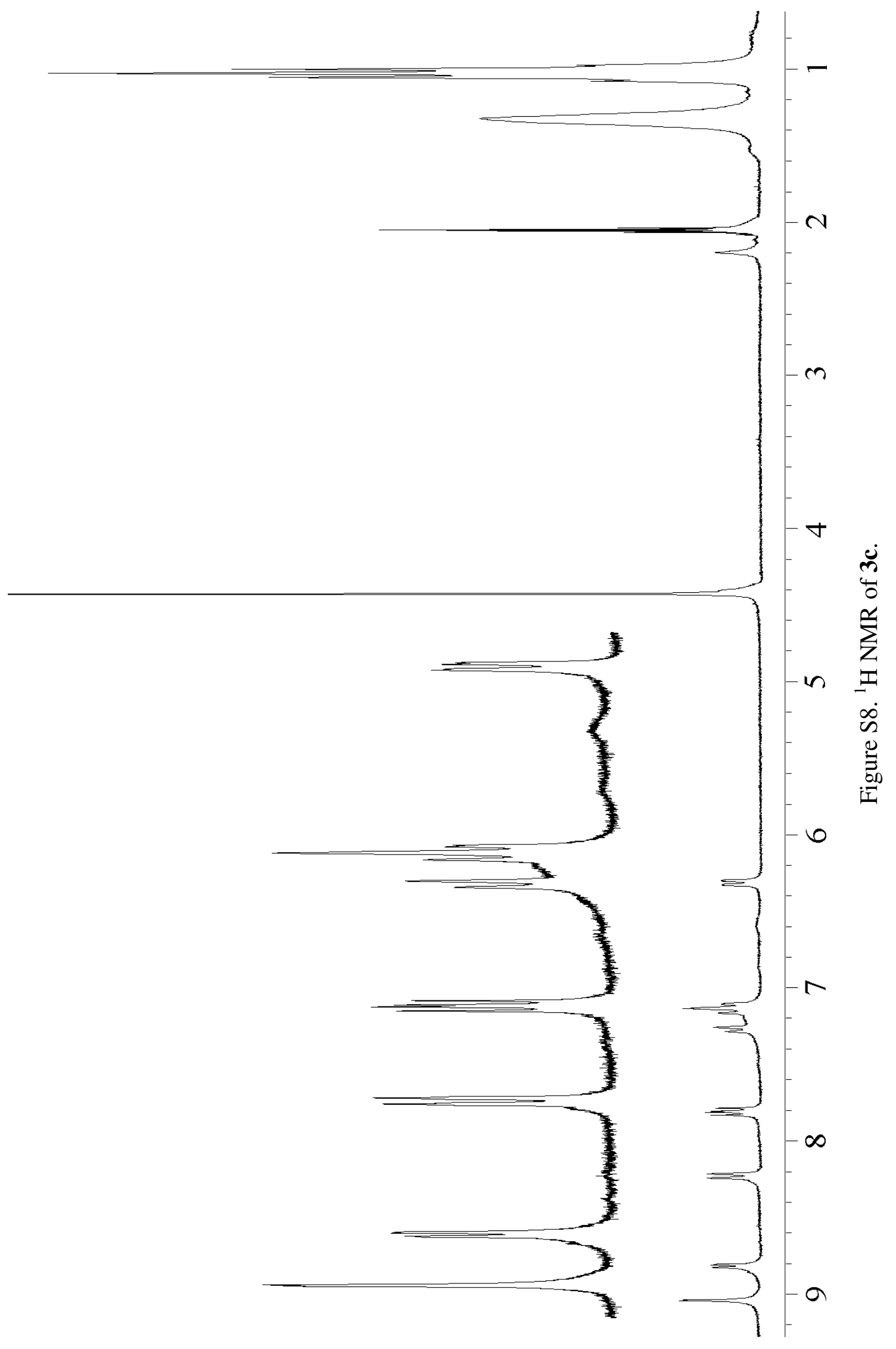




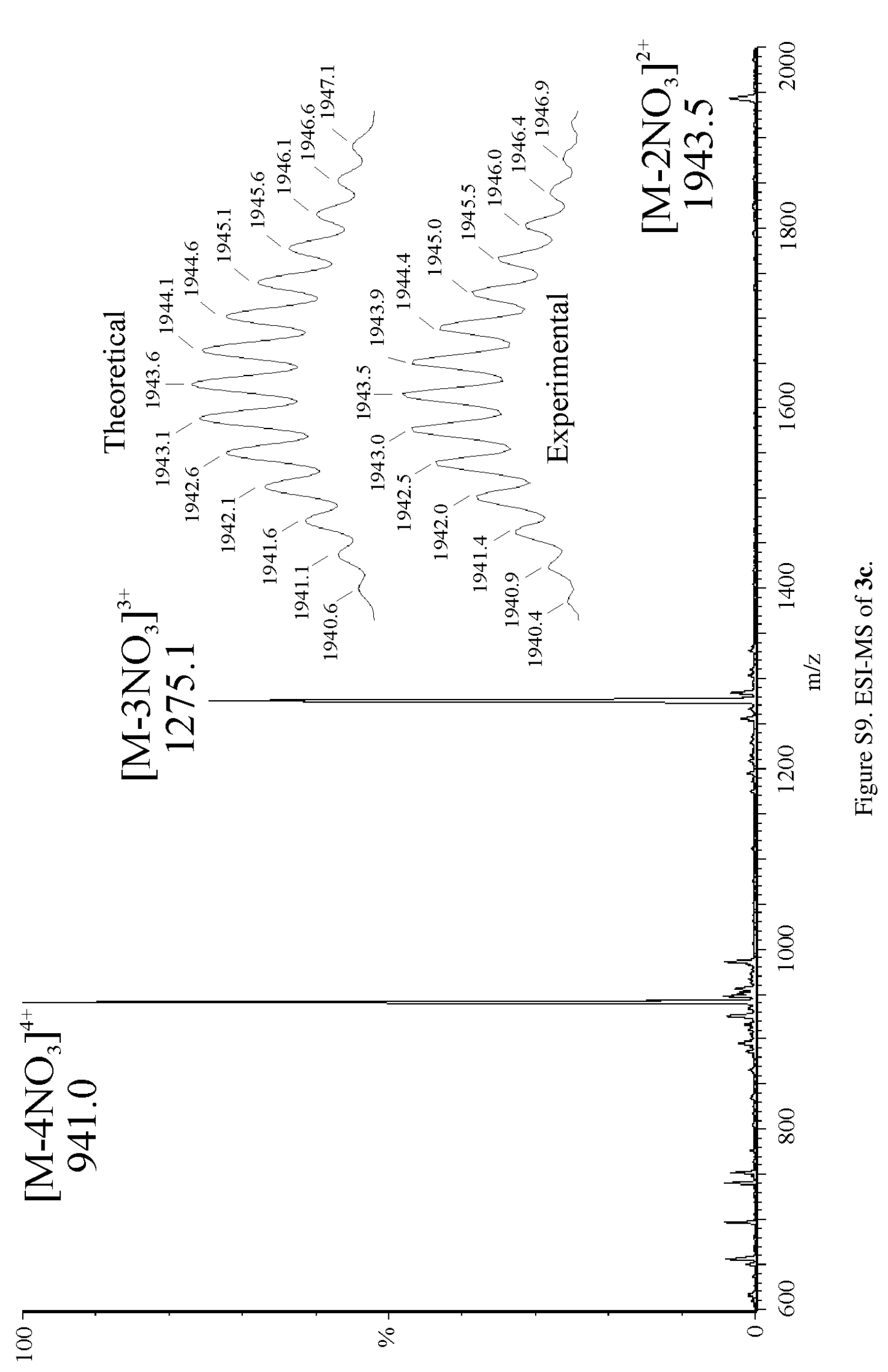




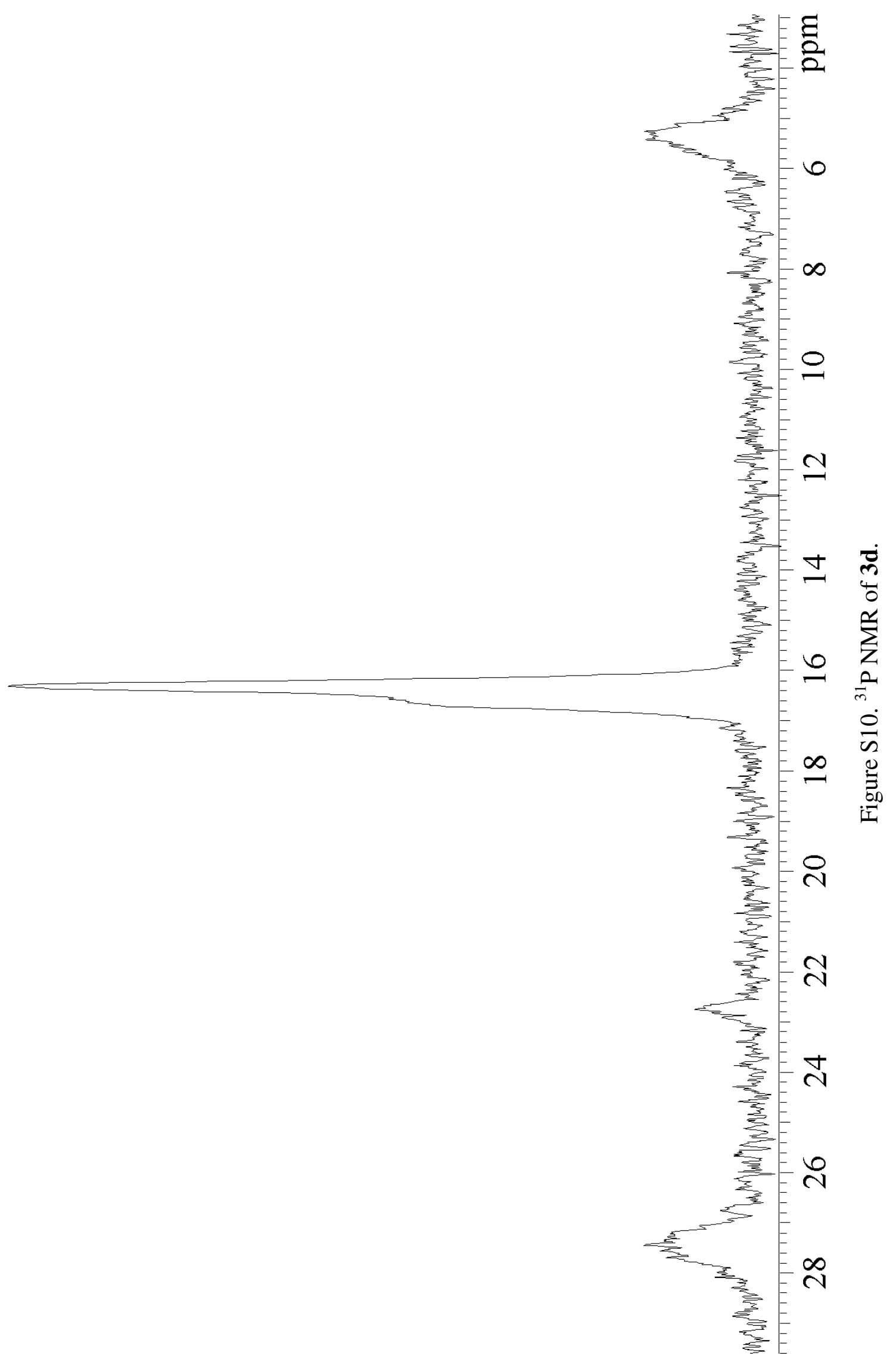









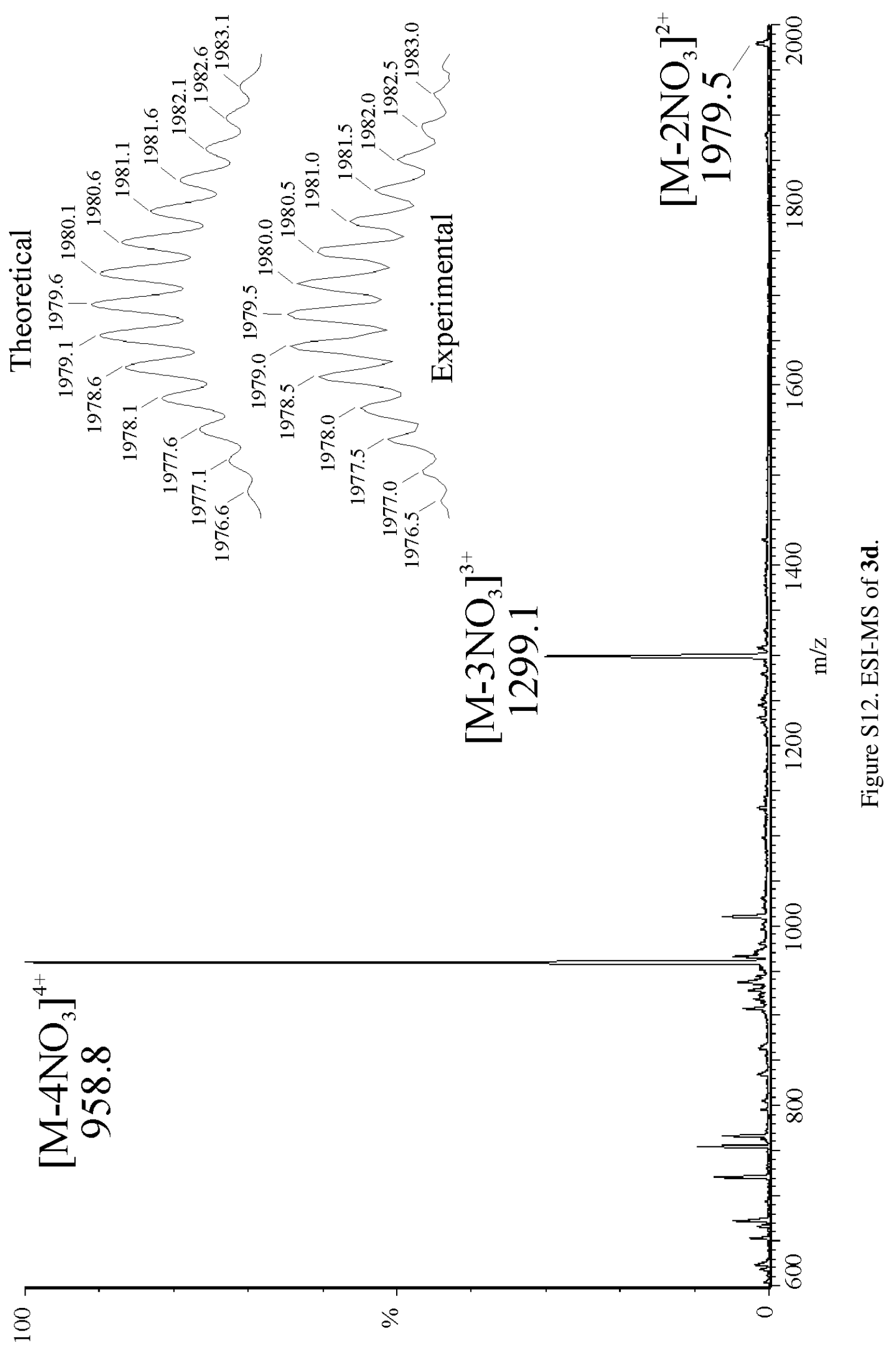

is 
$\frac{6}{n}$

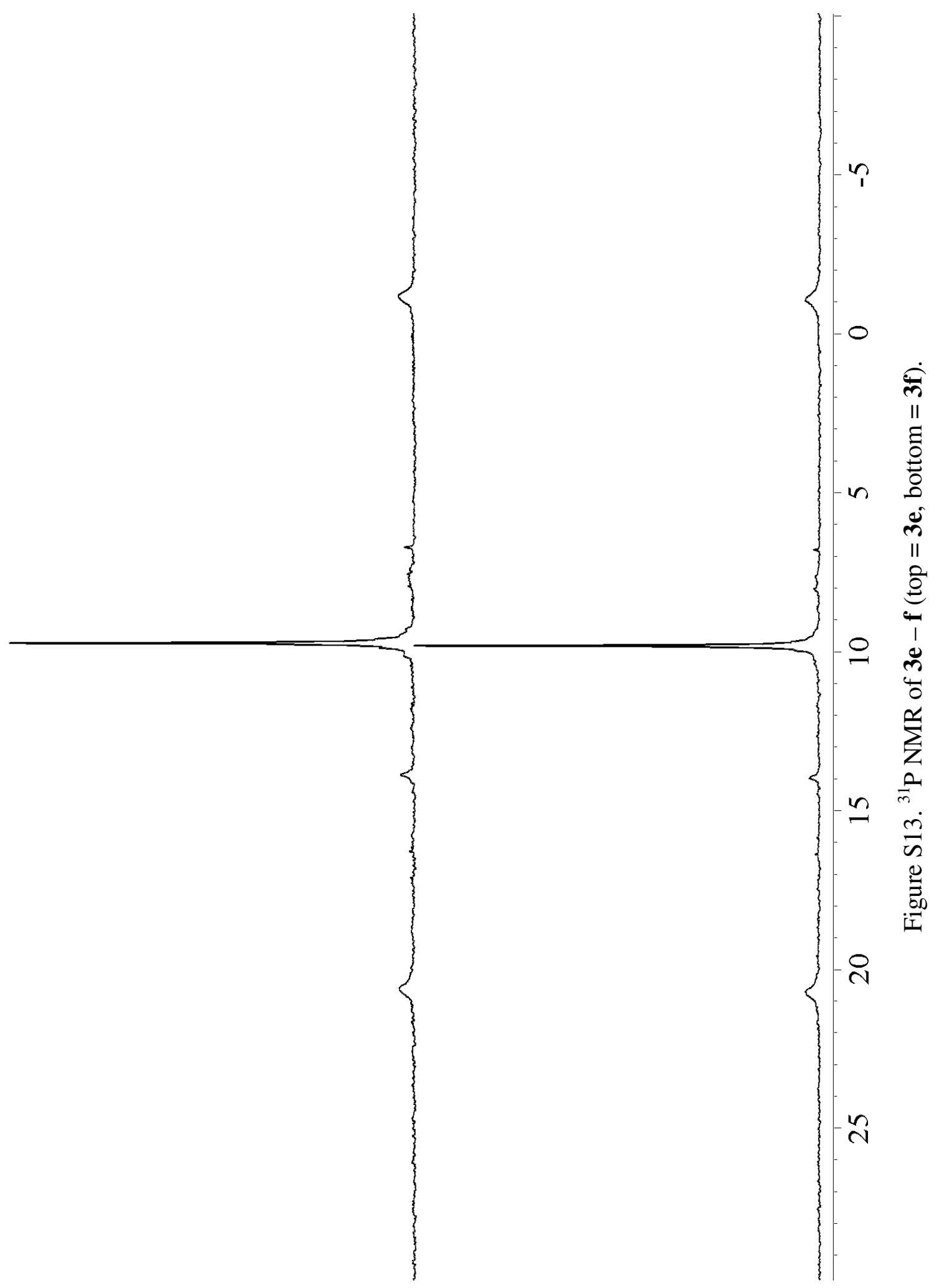


근

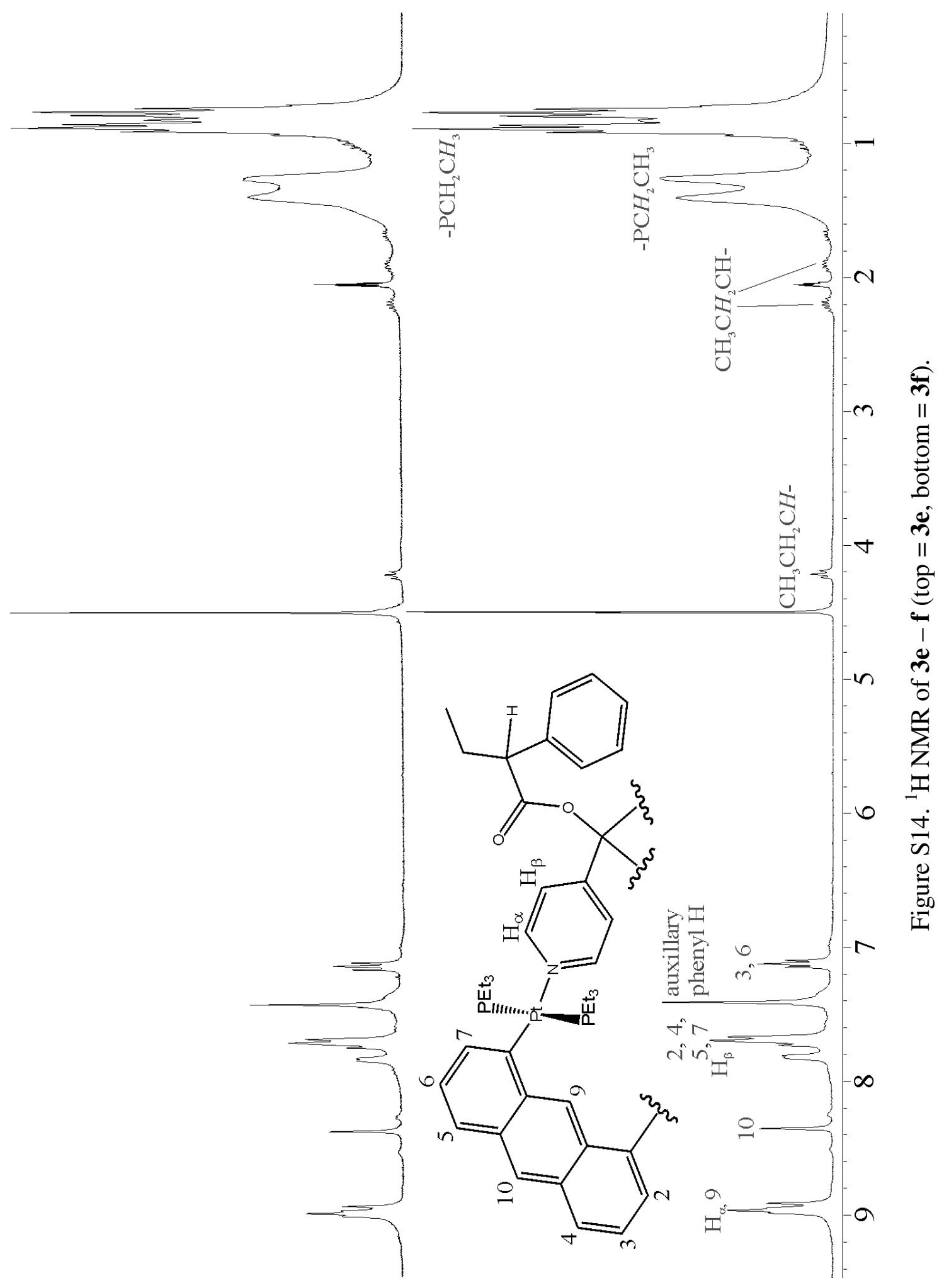




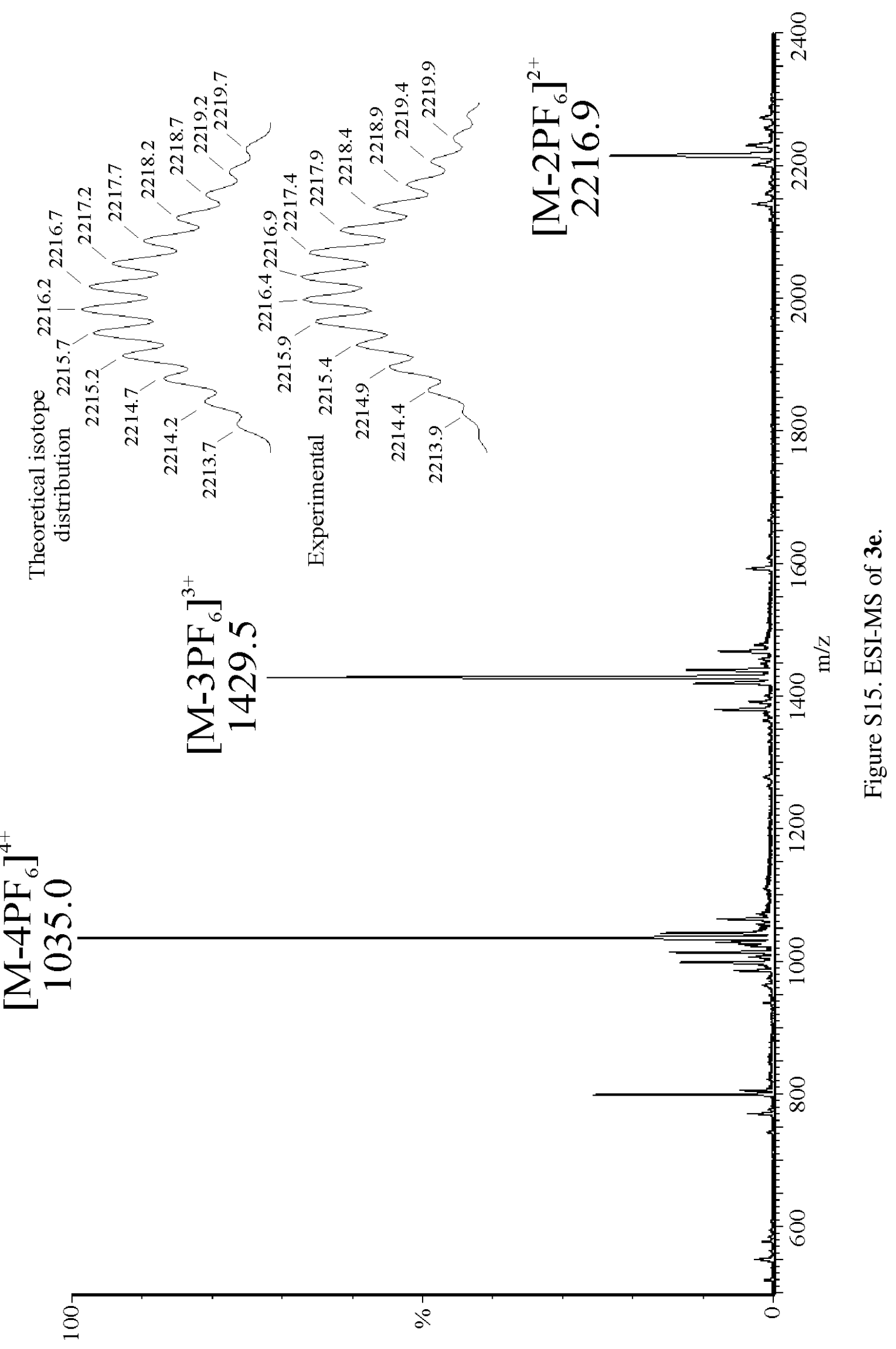

